\title{
Assembly of Model Membrane Nanodiscs for Native Mass Spectrome-
} try

\author{
Marius M. Kostelic, ${ }^{1}$ Ciara K. Zak, ${ }^{1}$ Hiruni S. Jayasekera, ${ }^{1}$ and Michael T. Marty ${ }^{1,2, *}$ \\ ${ }^{1}$ Department of Chemistry and Biochemistry and 2Bio5 Institute, University of Arizona, Tucson, AZ 85721, USA \\ KEYWORDS: Nanodiscs, lipids, native mass spectrometry
}

\begin{abstract}
Native mass spectrometry (MS) with nanodiscs is a promising technique for characterizing membrane protein and peptide interactions in lipid bilayers. However, prior studies have used nanodiscs made of only one or two lipids, which lack the complexity of a natural lipid bilayer. To better model specific biological membranes, we developed model mammalian, bacterial, and mitochondrial nanodiscs with up to four different phospholipids. Careful selection of lipids with similar masses that balance the fluidity and curvature enabled these complex nanodiscs to be assembled and resolved with native MS. We then applied this approach to characterize the specificity and incorporation of LL-37, a human antimicrobial peptide, in single lipid nanodiscs versus model bacterial nanodiscs. Overall, development of these model membrane nanodiscs reveals new insights into the assembly of complex nanodiscs and provides a useful toolkit for studying membrane protein, peptide, and lipid interactions in model biological membranes.
\end{abstract}

\section{INTRODUCTION:}

Native mass spectrometry (MS) has become a versatile tool for measuring protein-lipid interactions. ${ }^{1}$ Conventionally, detergents are used to solubilize membrane proteins for native MS. ${ }^{1-4}$ However, detergents do not necessarily replicate a natural lipid bilayer and can disrupt the structure and function of some complexes. ${ }^{5-7}$ Therefore, lipid bilayer mimetics have been developed to study membrane interactions in more natural environments. ${ }^{8}$

Nanodiscs have emerged as a promising alternative to detergents because they provide a controllable lipid bilayer and are relatively monodisperse. ${ }^{9}$ Native MS studies with nanodiscs have uniquely enabled the investigation of membrane protein oligomers within intact lipid bilayers. ${ }^{10-11}$ Nanodiscs have also proven useful for studying the mechanism of membrane-active peptides with native MS. ${ }^{12-14}$

Although nanodiscs can be formed with natural lipid extracts, ${ }^{15}$ most native MS experiments have used nanodiscs made of a single synthetic phospholipid because they produce well-resolved spectra. There is an intrinsic distribution in the number of lipids per nanodisc, so spectra show a regular series of peaks separated by the mass of the lipid. ${ }^{16-17}$ Adding a new lipid to the nanodisc adds another layer of complexity that makes resolution difficult. For example, Klassen and coworkers first employed mixed lipid nanodiscs that were made with glycolipids and phosphatidylcholine (PC), but the intact nanodiscs were unresolvable because of the complex lipid masses. ${ }^{18-20}$

By choosing two synthetic lipids with similar mass, native MS can resolve mixed lipid nanodiscs and determine the relative ratios of each species from the average mass of the lipid.21-22 Because these lipids have similar masses, nanodiscs with different numbers of each lipid have overlapping masses that form a clean series of peaks separated by the weighted average mass of the two lipids. These nanodiscs with binary mixtures of similar lipids have been used to understand how membrane proteins remodel their surrounding lipid environment. ${ }^{23}$ Building on this initial work, we recently developed a mass resonance approach to resolve nanodiscs containing two lipid types with very different masses. ${ }^{24}$ As long as the mass of one lipid was close to a simple fraction or an integer multiple of the second lipid, we were able to resolve the intact nanodiscs and characterize the stability of these mixed lipid nanodiscs. This method extended the types of lipids amenable for native MS of nanodiscs.

Here, we expand these approaches to develop mixed lipid nanodiscs suitable for native MS that model natural lipid bilayers. Biological membranes are difficult to mimic because they have a complex mixture of thousands of lipids. ${ }^{25}$ ${ }^{26}$ The wide diversity of masses prevents the resolution of nanodiscs formed with natural lipid extracts by native MS, and natural lipid extracts also do not provide the ability to control the ratios of different lipids. To overcome these limitations, we carefully chose sets of up to four different phospholipids with different head groups but similar masses, which allowed these model membrane nanodiscs to be resolved with native MS. We also observed some failed combinations that formed nanodiscs with three lipoprotein belts. Finally, we tested how these new lipid mixtures affect the incorporation of an antimicrobial peptide (AMP) LL-37 into nanodiscs. ${ }^{27}$ Ultimately, these controllable lipid mixtures will be useful for measuring polydisperse lipid interactions by native MS and exploring how the lipid bilayer environment affects membrane proteins and transmembrane peptides.

\section{METHODS:}

Nanodisc Preparation. 1-palmitoyl-2-oleoyl-sn-glycero-3-phosphocholine (POPC); 1-palmitoyl-2-oleoyl-sn- 
glycero-3-phosphoglycerol (POPG); 1-stearoyl-2-oleoyl-snglycero-3-phosphoethanolamine (SOPE); 1-palmitoyl-2oleoyl-sn-glycero-3-phospho-L-serine (POPS); N-stearoylD-erythro-sphingosylphosphorylcholine (SSM); 1,2-dipalmitoyl-sn-glycero-3-phosphocholine (DPPC); 2-dipalmitoyl-sn-glycero-3-phospho-(1'-rac-glycerol) (DPPG) 1palmitoyl-2-oleoyl-sn-glycero-3-phosphoethanolamine (POPE); 1,2-distearoyl-sn-glycero-3-phosphoethanolamine (DSPE); 1',3'-bis[1-palmitoyl-2-oleoyl-sn-glycero-3-phospho]-glycerol (POCL); 1',3'-bis[1,2-dioleoyl-sn-glycero-3phospho]-glycerol (TOCL); and 1',3'-bis[1,2-distearoyl-snglycero-3-phospho]-glycerol (TSCL) were purchased from Avanti Polar Lipids. Additional details on materials and membrane scaffold protein (MSP), MSP1D1(-), preparation are provided in the Supporting Information. Single lipid nanodiscs - made only of POPC, DPPC, or DPPG - were prepared as previously described. ${ }^{24,28}$ For mixed lipid nanodiscs, individual lipid stocks were dissolved in chloroform. To improve solubility, a 5:1 chloroform:methanol mixture was used to solubilize DPPC, DPPG, TSCL, and POCL. The desired lipid stocks were mixed prior to drying under nitrogen to remove the organic solvents. Drying multiple lipids together in chloroform allowed them to be resolubilized as a mixed membrane with the addition of detergent solution. ${ }^{15}$ For proper nanodisc assembly, we discovered that mixed lipid chloroform solutions needed to be clear and fully solubilized prior to evaporation. For DPPG- and DPPC-based nanodiscs, lipids in chloroform usually required heat (around $60{ }^{\circ} \mathrm{C}$ ) to fully solubilize. ${ }^{28}$ POPC/SOPE, DPPC/SOPE, and DPPG/SOPE mixtures were prepared at molar ratios of 75/25. POPC/SOPE/POPS and DPPC/SOPE/POPS compositions were made at a molar ratio of 65/25/10. POPC/SOPE/POPS/SSM and DPPC/SOPE/POPS/SSM nanodiscs were made at a ratio of 55/25/10/10. DPPG/SOPE/POCL and DPPC/SOPE/TSCL nanodiscs were prepared at a $65 / 25 / 5$ molar ratio.

After drying, lipids were resolubilized with $0.1 \mathrm{M}$ sodium cholate detergent. MSP1D1(-) was then added to the resuspended lipids in detergent. Optimal lipid ratios were determined empirically by testing several ratios and finding those that formed the most stable and monodisperse nanodiscs. For POPC nanodiscs, MSP was added at a 1:65, MSP:lipid molar ratio. For POPC-based mixed lipid compositions, MSP was added at a 1:80 MSP:lipid molar ratio. For DPPC and DPPG single and mixed lipid nanodiscs, MSP was added at a 1:110 MSP:lipid ratio. POPC-based nanodisc mixtures with lipids, cholate, and MSP were first incubated at 4 ${ }^{0} \mathrm{C}$ for 30 minutes. Amberlite XAD-2 beads were adjusted to match the reconstitution temperature and then added at a ratio of roughly 0.5 grams of beads per $1 \mathrm{~mL}$ volume. POPCbased reconstitution mixtures were then incubated with beads at $4{ }^{\circ} \mathrm{C}$ for at least 12 hours. Similarly, DPPC- and DPPG-based nanodisc mixtures were first incubated at 41 ${ }^{0} \mathrm{C}$ for 30 minutes prior to the addition of beads and incubation at $41{ }^{\circ} \mathrm{C}$ for 4 hours. For DPPG-based nanodiscs, 12 hour or longer incubations with beads were used to improve nanodisc quality. Following removal of beads by filtration, nanodiscs were purified using a Superose 6 Increase 10/300 column (GE Healthcare) into $0.2 \mathrm{M}$ ammonium acetate at $\mathrm{pH}$ 6.8. Final concentrations of nanodiscs following fraction collection varied from 2-10 $\mu \mathrm{M}$.

Peptide Nanodisc Preparation. Peptide nanodiscs were prepared as previously described. ${ }^{12-13}$ After the nanodiscs were purified, they were diluted to $2.5 \mu \mathrm{M}$. LL-37 was dissolved in methanol and then added to DPPG or DPPG/SOPE/POCL nanodiscs at a molar ratio of 3:1 LL-37: nanodisc using Hamilton syringes. Finally, imidazole was added to peptide nanodiscs to stabilize them during ionization. Imidazole was prepared in water at a concentration of $400 \mathrm{mM}$ with a $\mathrm{pH}$ of 6.8 . The final volume composition of peptide nanodisc mixtures were $19 \mu \mathrm{L}$ of $2.5 \mu \mathrm{M}$ nanodisc, $1.5 \mu \mathrm{L}$ of $400 \mathrm{mM}$ imidazole, and $3 \mu \mathrm{L}$ of $50 \mu \mathrm{M}$ peptide. The final concentration of nanodiscs was $2 \mu \mathrm{M}$, imidazole was $25 \mathrm{mM}$, and peptide was $6.38 \mu \mathrm{M}$. For control replicates without peptide, $3 \mu \mathrm{L}$ of methanol was added instead.

Mass Spectrometry. Details on mass spectrometry analysis are provided in the Supporting Information. Native mass spectra were analyzed using UniDec and MetaUniDec software as previously described.12,24,29-30 The mass range was set from 20-250 kDa with a charge range of 5-25. The peak width was set to $5 \mathrm{~m} / \mathrm{z}$. For peptide-nanodisc spectra, the peak width was set to $1 \mathrm{~m} / \mathrm{z}$. The charge smooth width was -0.5 , and the mass smooth width was 1 with the theoretical average lipid mass of the mixture used as the mass difference. Average lipid mass analysis was used to confirm the overall lipid composition in mixed lipid nanodiscs. ${ }^{23}$ For peptide-nanodisc experiments, mass defect analysis was used to determine the stoichiometry of LL-37 in DPPG and DPPG/SOPE/POCL nanodiscs as previously described.12-13 Further details on data analysis are provided in the Supporting Methods.

\section{RESULTS:}

Our overall goal was to create nanodiscs that modeled the phospholipid compositions of mammalian cells, bacteria, and mitochondria but would be suitable for native MS analysis. Unfortunately, nanodiscs formed with natural lipid extracts are too heterogeneous for native MS. Moreover, the lipid composition cannot be easily adjusted with natural lipid mixtures to test the influence of specific lipids. Thus, we sought to model natural lipid membranes by strategically choosing synthetic lipids that represent common head groups in each of these membranes but have similar masses. We have previously applied this approach to study binary mixtures of several different lipid types. ${ }^{22,24}$ Here, we extended this approach to create resolvable nanodiscs with up to four different phospholipids.

Model Mammalian Nanodiscs. Our first goal was to create nanodiscs that mimic a mammalian phospholipid composition yet could be resolved by native MS. ${ }^{26,31}$ Mammalian membranes are highly complex with thousands of different lipid species. ${ }^{25,31-32}$ To simplify the model, we chose four common phospholipid classes: PC, phosphatidylethanolamine (PE), phosphatidylserine (PS), and sphingomyelin (SM).

For model mammalian nanodiscs, we chose palmitoyloleoyl-phosphatidylcholine (POPC) as the main eukaryotic 

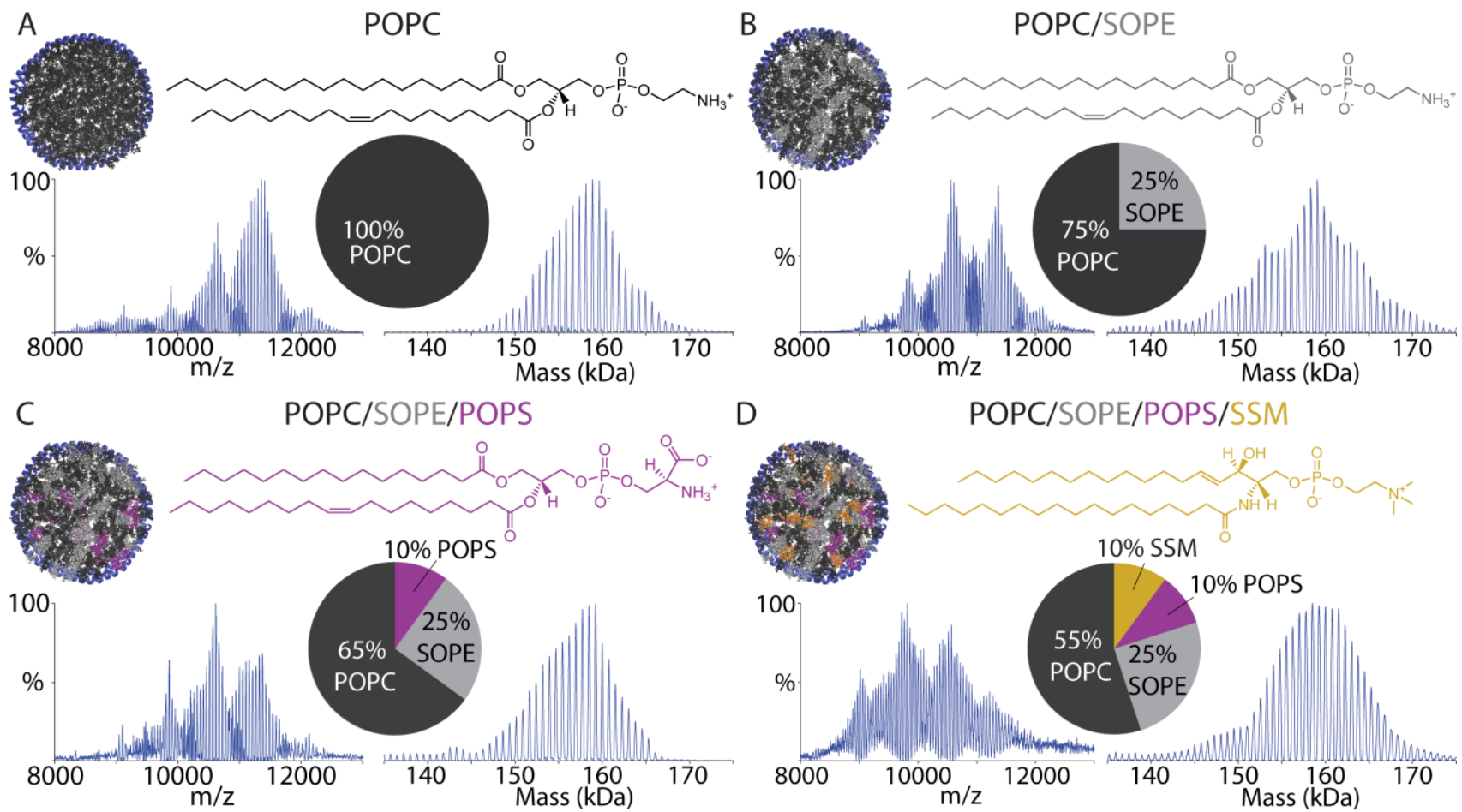

Figure 1. Native MS of model mammalian nanodiscs in positive ionization mode with 2-methylimidazole with corresponding raw mass spectra to the left, deconvolved mass spectra to the right, simulated nanodiscs with color-coordinated lipids, and pie charts showing percent compositions. A) POPC nanodiscs with the POPC lipid structure in black. B) POPC/SOPE nanodiscs with the SOPE lipid structure shown in grey. C) POPC/SOPE/POPS nanodiscs with the POPS structure shown in magenta. D) POPC/SOPE/POPS/SSM nanodiscs with the SSM structure shown in gold.

phospholipid due to its abundance in mammalian cell membranes. ${ }^{33}$ We then sought to incorporate PE, which is often the second most abundant phospholipid class in mammalian membranes. ${ }^{33}$ Incorporation of PE lipids is challenging because PE has a negative curvature that may perturb the assembly of flat nanodiscs. ${ }^{34}$ To overcome challenges in assembling POPC/PE nanodiscs, we screened several PE phospholipids. We discovered that stearyl-oleoyl-phosphatidylethanolamine (SOPE) formed the most stable and consistent nanodiscs. Moreover, because its mass is close to POPC, it provided well-resolved spectra. However, at higher collision voltages, SOPE was preferentially ejected from POPC/SOPE nanodiscs until the only remaining lipid present was POPC (Figure S1). To avoid this preferential dissociation, we tested imidazole derivatives that have previously been shown to stabilize nanodisc complexes. ${ }^{35} \mathrm{We}$ found that 2-methylimidazole stabilized POPC/SOPE nanodiscs the most and caused POPC/SOPE nanodiscs to have higher mass distributions, indicating less dissociation (Figure 1). Interestingly, the average lipid mass shows that 2methylimidazole retained the labile SOPE up to $200 \mathrm{~V}$ applied in the HCD cell (Figure S1). Because 2-methylimidazole stabilized POPC-based mixed lipid nanodiscs the most, all sequential POPC-based nanodiscs were analyzed with 2methylimidazole.

With 2-methylimidazole, nanodiscs formed at a 75/25 POPC/SOPE ratio showed an overall mass distribution and polydispersity similar to POPC nanodiscs (Figure 1A, B).
The difference between POPC and POPC/SOPE nanodiscs can be seen by comparing the average mass of the lipids. POPC nanodiscs have an average lipid mass of $760.24 \pm 0.03$ $\mathrm{Da}$, which is close to the expected mass of POPC (760.08 Da). Because SOPE is lighter (746.05 Da), the average lipid mass of POPC/SOPE nanodiscs was lower, showing an average lipid mass of $757.02 \pm 0.29 \mathrm{Da}$, close to the expected value of 756.57 Da for a 75/25 mixture of POPC/SOPE. Predicted and calculated average lipid masses for each mixed lipid nanodisc are shown in Table S1.

Building on the stable POPC/SOPE nanodiscs, we incorporated additional phospholipids to increase the complexity of the model mammalian membrane. Phosphatidylserine (PS) is the most abundant anionic lipid in eukaryotic membranes and is vital for cell survival and signaling. ${ }^{36-37} \mathrm{We}$ chose to incorporate POPS because its mass (762.00 Da) is close to POPC (760.08 Da) and SOPE (746.05 Da). Using 2methylimidazole to stabilize the complex during ionization, we created three-component nanodiscs with a 65/25/10 ratio of POPC/SOPE/POPS that were stable and had an average lipid mass of 757.28 \pm 0.20 Da (Figure 1C). Although the average lipid masses of POPC/SOPE nanodiscs and POPC/SOPE/POPS nanodiscs are too close to differentiate, small molecule MS confirmed incorporation of POPS (Figure S2).

Finally, we incorporated SSM as the fourth component of our model mammalian nanodiscs. Sphingomyelin is important for signal transduction and is relatively abundant in 

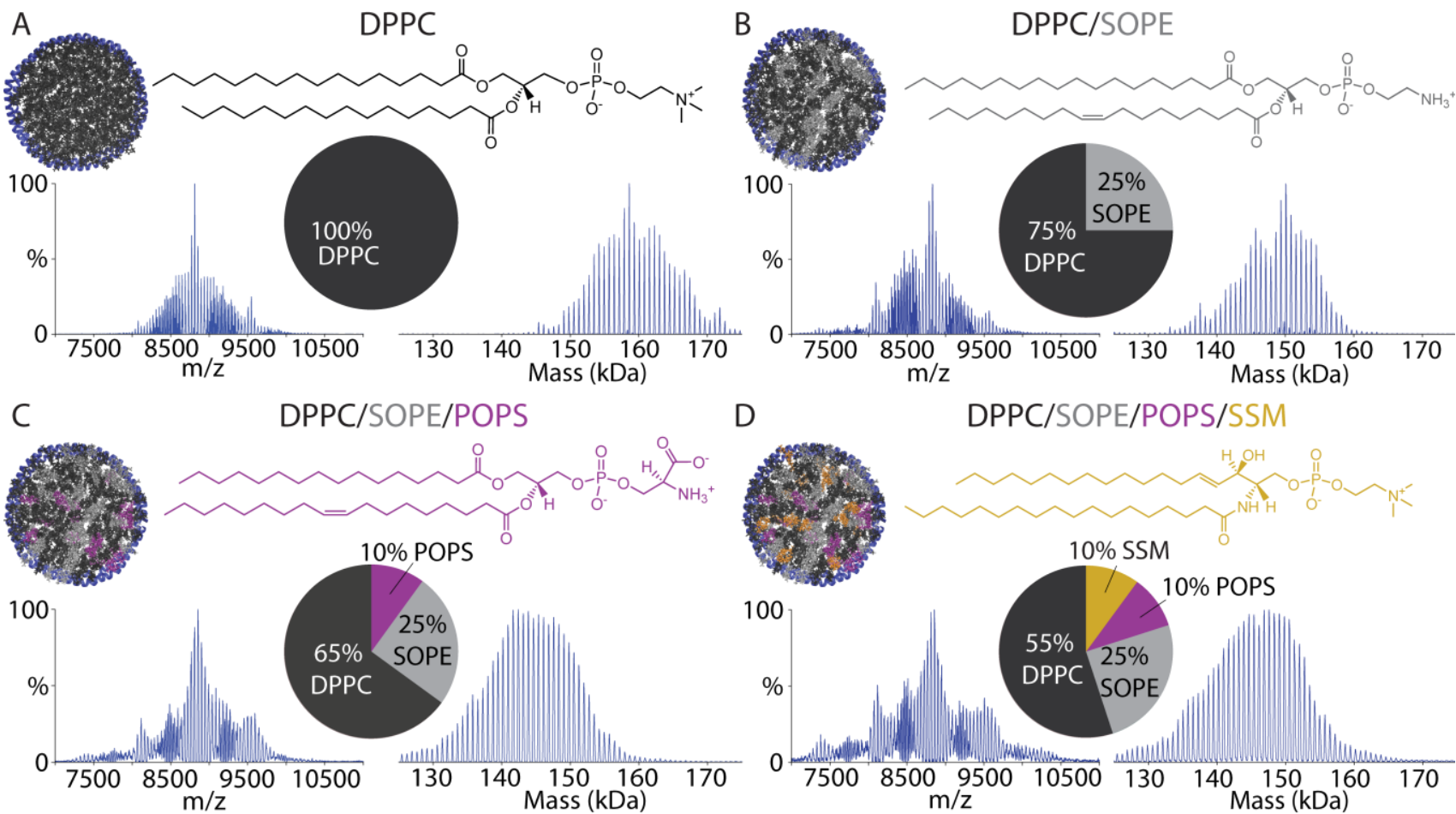

Figure 1. Native MS of saturated model mammalian nanodiscs in positive mode with imidazole. For each section: raw mass spectra are shown to the left, deconvolved mass spectra to the right, simulated nanodiscs with color-coordinated lipids, and pie charts showing percent composition. A) DPPC nanodiscs with the DPPC lipid structure is shown in black. B) DPPC/SOPE nanodiscs with the SOPE lipid structure shown in grey. C) DPPC/SOPE/POPS nanodiscs with the POPS structure shown in magenta. D) DPPC/SOPE/POPS/SSM nanodiscs with the SSM structure shown in gold.

mammalian membranes. ${ }^{38-40}$ Nanodiscs with a ratio of 55/25/10/10 POPC/SOPE/POPS/SSM gave well-resolved spectra and had an average lipid mass of $753.13 \pm 0.86 \mathrm{Da}$, which matched the expected 753.86 Da (Figure 1D). Overall, model mammalian nanodiscs with these lipids were stable and showed well resolved spectra. We expect that additional lipids could be easily incorporated to this mixture, provided they are similar in mass to POPC.

Saturated Model Mammalian Nanodiscs. To extend this mixed lipid nanodisc recipe to saturated lipids, we sought to create saturated model mammalian nanodiscs using dipalmitoyl-phosphatidylcholine (DPPC) as the base lipid. Like POPC-based nanodiscs, we first incorporated PE. Initially, we tried to incorporate distearoyl-phosphatidylethanolamine (DSPE) into DPPC nanodiscs to maintain saturated lipid tails with a mass close to DPPC. However, DPPC/DSPE mixtures were often insoluble and not amenable to nanodisc formation. We next tested SOPE, and we screened different MSP:lipid ratios, incubation times, and assembly temperatures. Similar to prior studies, it was best to assemble nanodiscs at the phase transition temperature of the most abundant lipid, which is $41^{\circ} \mathrm{C}$ for DPPC. ${ }^{15}$ After optimizing the ratios and assembly times, we were able to prepare reproducible DPPC/SOPE nanodiscs at a molar ratio of 75/25 (Figure 2).

Like POPC/SOPE nanodiscs, DPPC/SOPE nanodiscs showed significant dissociation in positive ionization mode. At high collision voltages, SOPE was preferentially ejected from the nanodisc until the only lipid left was DPPC. Unlike the POPC-based nanodiscs, 2-methylimidazole did not stabilize DPPC/SOPE nanodiscs more than imidazole. Thus, we used imidazole to stabilize DPPC/SOPE nanodiscs. DPPC only nanodiscs with imidazole showed an average lipid mass of $734.17 \pm 0.07 \mathrm{Da}$, which was very close to the mass of DPPC (734.04 Da). DPPC/SOPE nanodiscs had an average lipid mass of $736.16 \pm 0.05 \mathrm{Da}$, which was less than the expected 737.04 $\mathrm{Da}$ and indicated a slight enrichment of DPPC.

We then incorporated POPS into DPPC/SOPE nanodiscs to match our previous model mammalian nanodiscs. DPPC/SOPE/POPS nanodiscs with a molar ratio of $65 / 25 / 10$ were well resolved in positive mode with imidazole and had an average lipid mass of $739.25 \pm 0.76 \mathrm{Da}$, which matched the expected average mass of 739.84 Da (Figure 2C). Finally, we incorporated SSM to assemble DPPC/SOPE/POPS/SSM nanodiscs at a molar ratio of 55/25/10/10. DPPC/SOPE/POPS/SSM nanodiscs were well resolved in positive ionization mode with imidazole (Figure 2D) and had an average lipid mass of 739.84 $\pm 0.49 \mathrm{Da}$, which matched the expected value of $739.54 \mathrm{Da}$. Small molecule MS confirmed the incorporation of SSM into DPPC/SOPE/POPS nanodiscs (Figure S2). Like the POPCbased model mammalian nanodiscs, incorporating phospholipids other than PE was less difficult than optimizing the DPPC/SOPE mixture. Interestingly, DPPC-based mixed lipid nanodiscs have lower intact mass distributions than DPPC-only nanodiscs (Figure 2), which shows that 
incorporation of the unsaturated lipids affects the overall lipid packing within the nanodisc.

Model Bacterial Nanodiscs. We next sought to create model bacterial nanodiscs to complement our model mammalian nanodiscs. Bacterial membranes are challenging to mimic due to their wide diversity in lipid structures. ${ }^{41}$ For simplicity, we focused on typical bacterial phospholipids with phosphatidylglycerol (PG), PE, and cardiolipin (CL). We chose PG as the main bacterial lipid because of its prevalence in gram-negative and gram-positive bacteria. ${ }^{41}$

We initially tested palmitoyl-oleoyl-phosphatidylglycerol (POPG) as the main bacterial phospholipid to match the tails of our POPC-based model mammalian nanodiscs. We first attempted to incorporate PE-which is equally or more abundant than PG in bacterial membranes-into POPG nanodiscs. ${ }^{41}$ We started with SOPE because it has a similar mass to POPG and worked well in model mammalian nanodiscs. However, POPG/SOPE nanodisc mixtures formed a higher mass distribution at 200-250 kDa with 3 MSP belts (Figure S3). These triple belt lipid nanoparticles had previously been seen but at much lower abundances in POPC/POPS and POPC/POPG nanodiscs. ${ }^{22}$ Regardless of the ratio, incubation time, and temperature of assembly, this higher mass distribution dominated the spectra. Similar problems were observed with palmitoyl-oleoyl-PE (POPE). Interestingly, the SEC profile was not significantly shiftedcompared to POPC/SOPE nanodiscs that formed
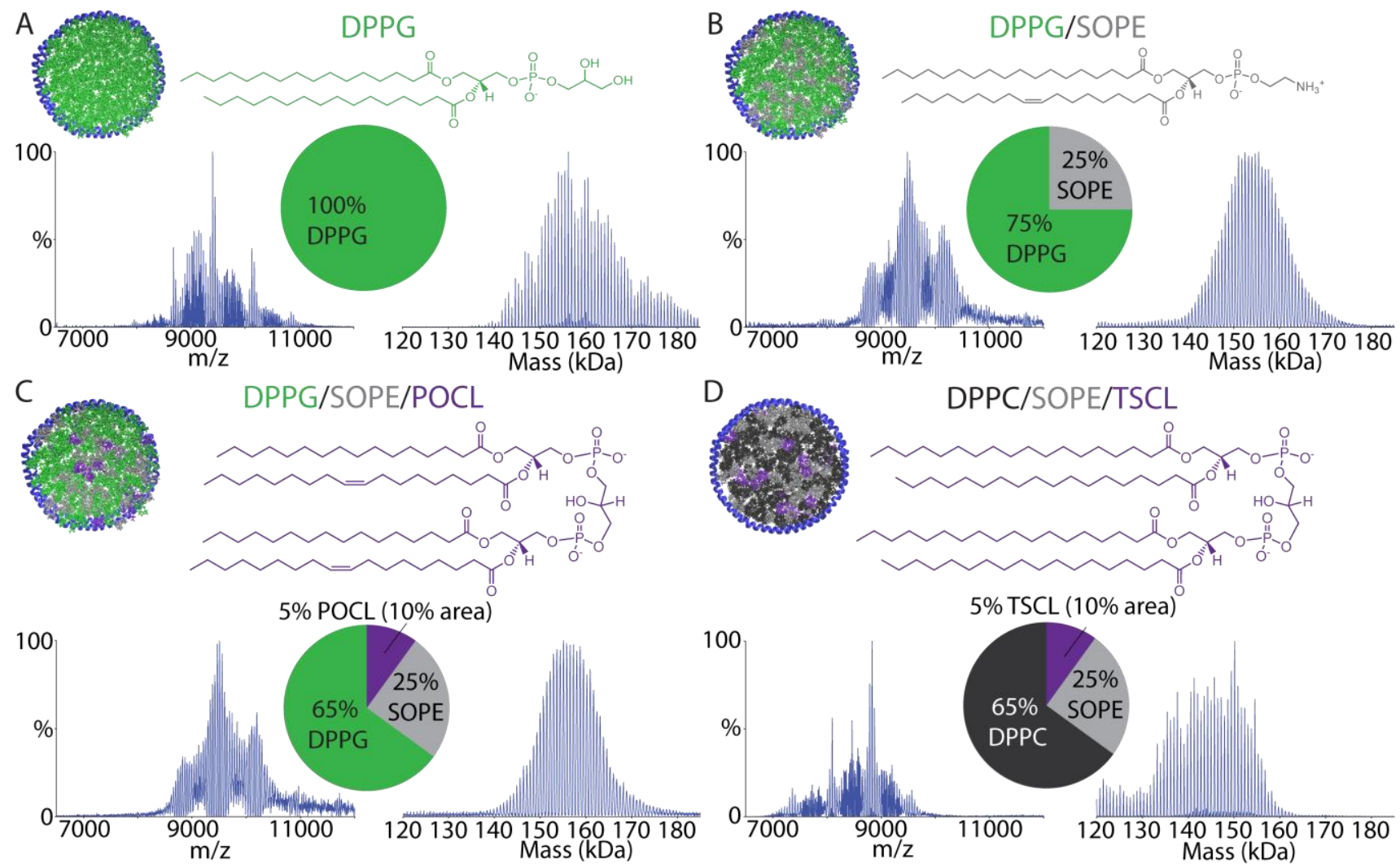

Figure 2. Native MS of model bacterial and mitochondrial nanodiscs in positive mode with imidazole. Each section shows the raw mass spectra on the left, the deconvolved mass spectra on the right, simulated mixed lipid nanodiscs with color coordinated lipids, and pie charts showing percent compositions. A) DPPG nanodiscs with the DPPG structure shown in green. B) DPPG/SOPE nanodiscs with the SOPE structure shown in grey. C) Model bacterial DPPG/SOPE/POCL nanodiscs with the POCL structure shown in purple. D) Model mitochondrial DPPC/SOPE/TSCL nanodiscs with the TSCL structure shown in purple. 
to incorporate a tetra-oleoyl-cardiolipin (TOCL), which has fully unsaturated tails. However, incorporating fully unsaturated cardiolipin into DPPG/SOPE nanodiscs caused the formation of more triple belt nanoparticles. To retain the conventional nanodisc structure for this mixture of lipids, we used a partially saturated cardiolipin palmitoyl-oleoyl-cardiolipin (POCL). We were able to assemble model bacterial DPPG/SOPE/POCL nanodiscs. DPPG/SOPE/POCL nanodiscs take advantage of the mass resonance between POCL and DPPG/SOPE lipids. ${ }^{24}$ Because the mass of POCL is roughly equal to two DPPG or SOPE lipids, peaks with different numbers of lipids overlap, and spectra are well resolved. We chose to create model bacterial nanodiscs at a molar ratio of 65/25/5 DPPG/SOPE/POCL rather than $65 / 25 / 10$ because POCL is twice the size of DPPG and SOPE. DPPG/SOPE/POCL nanodiscs have an average lipid mass of $725.54 \pm 0.42 \mathrm{Da}$, which was slightly less to the expected 726.74 Da (Figure 3C). Overall, these carefully selected lipids provide stable nanodiscs suitable for native MS that provide a simplified model of bacterial membranes.

Model Mitochondrial Nanodiscs. Mitochondria are crucial organelles for eukaryotic cells and have their own unique lipid membrane composition. ${ }^{31,45}$ Within mitochondrial membranes, particularly in the inner mitochondrial
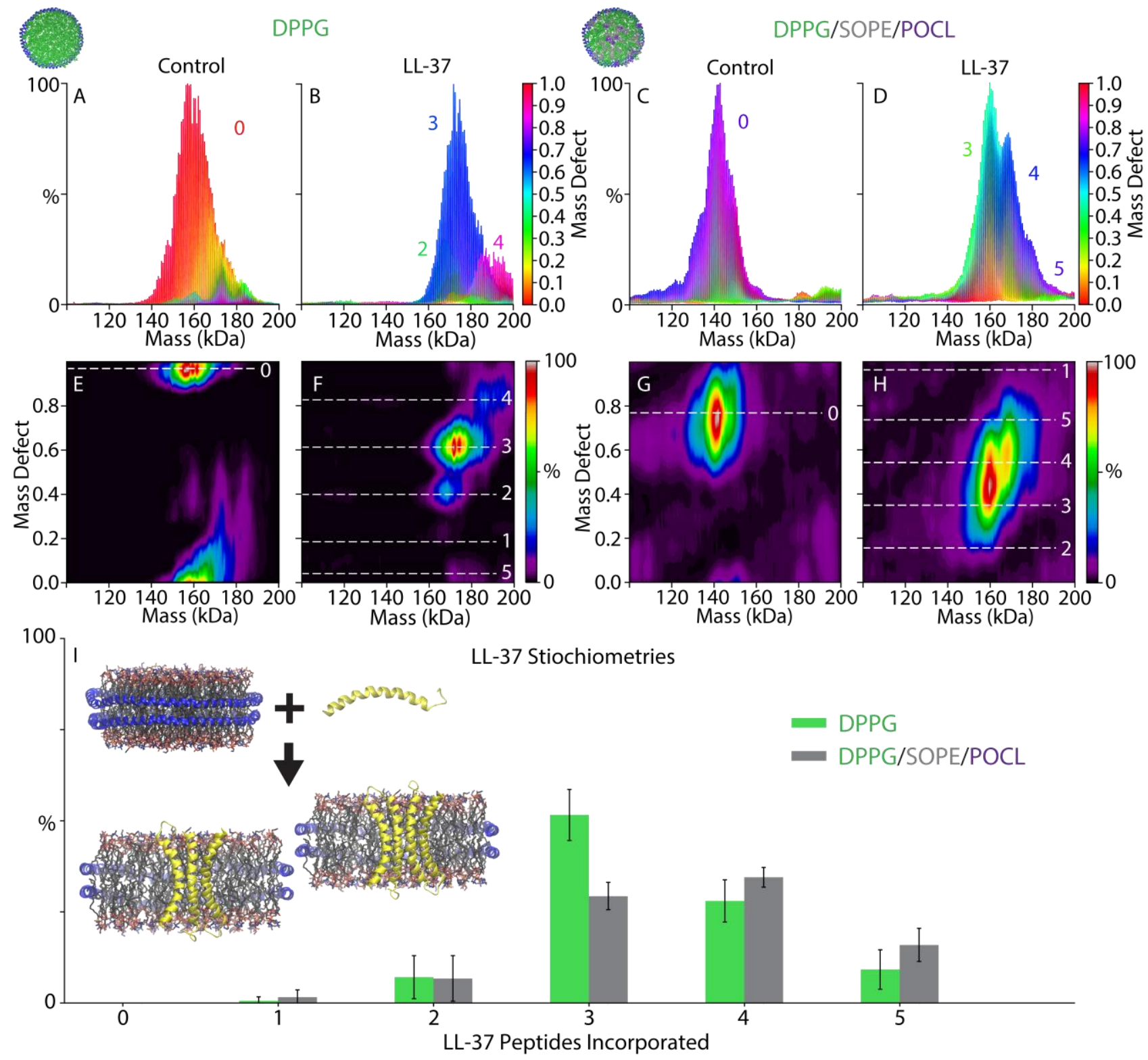

Figure 4. Incorporation of LL-37 in DPPG nanodiscs vs DPPG/SOPE/POCL nanodiscs. Deconvolved mass spectra of A) DPPG nanodiscs alone, B) 3:1 LL-37:DPPG nanodiscs, C) DPPG/SOPE/POCL nanodiscs alone, and D) 3:1 LL-37:DPPG/SOPE/POCL nanodiscs. Mass peaks are colored by mass defect with labeled stoichiometries. Corresponding mass defect plot with mass defect on the $y$-axis and mass on $x$-axis with predicted stoichiometries as dotted white lines for E) control DPPG nanodiscs, F) 3:1 LL-37:DPPG nanodiscs, G) control DPPG/SOPE/POCL nanodiscs, and H) 3:1 LL-37:DPPG/SOPE/POCL nanodiscs. I) Bar charts showing average relative intensities of LL-37 stoichiometries in nanodiscs with DPPG only (green) or DPPG/SOPE/POCL (grey). Error bars are shown as the standard deviation of three replicate nanodisc assemblies. LL-37 PDB:2K60. 
membrane (IMM), cardiolipin participates in several cellular functions such as energy transfer, redox signaling, and apoptosis. ${ }^{45-48}$ Thus, we sought to create model mitochondrial nanodiscs that match the IMM phospholipid composition, which is mostly $\mathrm{PC} / \mathrm{PE} /$ cardiolipin. ${ }^{31,49}$ To create model mitochondrial membranes, we substituted the DPPG used in our bacterial model with DPPC to create 65/25/5 DPPC/SOPE/POCL nanodiscs. However, DPPC/SOPE/POCL mixtures formed mostly higher mass, triple belt nanoparticles. To address this challenge, we tested tetra-stearoyl-cardiolipin (TSCL), a fully saturated cardiolipin with a mass close to two DPPC lipids. Model mitochondrial nanodiscs with a lipid composition of 65/25/5 DPPC/SOPE/TSCL provided well-resolved mass spectra (Figure 3D). Model mitochondrial nanodiscs were similarly stabilized by imidazole and had an average lipid mass of $736.46 \pm 0.05$ Da, which was slightly below the expected average lipid mass of 736.94 Da. Small molecule MS analysis confirmed incorporation of TSCL into DPPC/SOPE nanodiscs (Figure S2).

LL-37 in Model Bacterial Nanodiscs. LL-37 is a human antimicrobial peptide that plays an important role in the innate immune system by targeting and disrupting bacterial membranes. However, the mechanism of LL-37 activity and membrane interactions are unclear. ${ }^{50}$ LL-37 is thought to incorporate into bacterial membranes to form pores that lead to cell death, but the oligomeric state and assembly mechanisms of these pores are inconclusive. Recently, we discovered that LL-37 has partial specificity for DMPG (more bacterial) over DMPC (more mammalian) nanodiscs. ${ }^{12-13}$ We wanted to further understand how other bacterial phospholipids affect the incorporation of LL-37 in membranes. Thus, we compared the incorporation of LL-37 in DPPG nanodiscs to model bacterial (DPPG/SOPE/POCL) nanodiscs.

We found that LL-37 has a range of stoichiometries in DPPG nanodiscs but ultimately prefers a trimeric state at a 3:1 ratio of LL-37:nanodisc (Figure 4). Compared to DPPG nanodiscs, DPPG/SOPE/POCL nanodiscs show less LL-37 trimer and slightly more nanodiscs with four and five peptides incorporated (Figure 4I). However, the average incorporation of LL-37 between DPPG and model bacterial nanodiscs are similar. This indicates that PG is the main lipid component needed for LL-37 incorporation and oligomerization in these model bilayers, but other lipids play a subtle role in membrane interactions. Ultimately, these data show that model membrane nanodiscs can be used to gain stoichiometric information of incorporated proteins and peptides.

\section{CONCLUSIONS:}

By using lipids that are close in mass, we resolved nanodiscs that model mammalian, bacterial, and mitochondrial membranes with native MS. Interestingly, the proper formation of these mixed lipid nanodiscs required the right balance of saturation, tail length, and headgroup. We confirmed the incorporation of each lipid by observing the average lipid mass and presence of lipids in small molecule spectra. Finally, we measured the incorporation and specificity of LL-37 for DPPG nanodiscs and model bacterial nanodiscs. We found that LL-37 shows a similar but slightly higher incorporation in model bacterial nanodiscs.

Overall, these results establish methods of preparing and characterizing nanodiscs with complex lipid compositions with native MS. We expect that the foundation presented here will enable other lipids to be added to further increase the complexity of membranes for native MS, thereby enabling analysis of protein-lipid and peptide-lipid interactions in increasingly better models of their natural environments.

\section{ASSOCIATED CONTENT}

Supporting Information. The Supporting Information is available free of charge via the Internet at http://pubs.acs.org and includes supplemental methods, table, and figures.

\section{AUTHOR INFORMATION}

\section{Corresponding Author \\ *mtmarty@arizona.edu}

\section{ACKNOWLEDGMENT}

The pMSP1D1 plasmid was a gift from Stephen Sligar (Addgene plasmid \#20061). The authors thank Maria Reinhardt-Szyba, Kyle Fort, and Alexander Makarov at Thermo Fisher Scientific for their support on the UHMR Q-Exactive HF instrument. This work was funded by the National Science Foundation (CHE1845230).

\section{REFERENCES}

1. Keener, J. E.; Zhang, G.; Marty, M. T., Native Mass Spectrometry of Membrane Proteins. Anal. Chem. 2021, 93 (1), 583-597.

2. Dixit, S. M.; Polasky, D. A.; Ruotolo, B. T., Collision induced unfolding of isolated proteins in the gas phase: past, present, and future. Curr. Opin. Chem. Biol. 2018, 42, 93-100.

3. Gao, Y.; Cao, E.; Julius, D.; Cheng, Y., TRPV1 structures in nanodiscs reveal mechanisms of ligand and lipid action. Nature 2016, 534 (7607), 347-351.

4. Bolla, J. R.; Agasid, M. T.; Mehmood, S.; Robinson, C. V., Membrane Protein-Lipid Interactions Probed Using Mass Spectrometry. Annu. Rev. Biochem. 2019, 88, 85-111.

5. Guo, Y., Be Cautious with Crystal Structures of Membrane Proteins or Complexes Prepared in Detergents. Crystals 2020, 10 (2), 86.

6. Konijnenberg, A.; Yilmaz, D.; Ingolfsson, H. I.; Dimitrova, A.; Marrink, S. J.; Li, Z.; Venien-Bryan, C.; Sobott, F.; Kocer, A., Global structural changes of an ion channel during its gating are followed by ion mobility mass spectrometry. Proc. Natl. Acad. Sci. U. S. A. 2014, 111 (48), $17170-17175$.

7. Reading, E.; Walton, T. A.; Liko, I.; Marty, M. T.; Laganowsky, A.; Rees, D. C.; Robinson, C. V., The Effect of Detergent, Temperature, and Lipid on the Oligomeric State of MscL Constructs: Insights from Mass Spectrometry. Chem. Biol. 2015, 22 (5), 593-603.

8. Marty, M. T.; Hoi, K. K.; Robinson, C. V., Interfacing Membrane Mimetics with Mass Spectrometry. Acc. Chem. Res. 2016, 49 (11), 2459 2467.

9. Sligar, S. G.; Denisov, I. G., Nanodiscs: A toolkit for membrane protein science. Protein Sci. 2020, 10.1002/pro.3994.

10. Marty, M. T.; Hoi, K. K.; Gault, J.; Robinson, C. V., Probing the Lipid Annular Belt by Gas-Phase Dissociation of Membrane Proteins in Nanodiscs. Angew. Chem. Int. Ed. Engl. 2016, 55 (2), 550-554.

11. Keener, J. E.; Zambrano, D. E.; Zhang, G.; Zak, C. K.; Reid, D. J.; Deodhar, B. S.; Pemberton, J. E.; Prell, J. S.; Marty, M. T., Chemical Additives Enable Native Mass Spectrometry Measurement of Membrane Protein Oligomeric State within Intact Nanodiscs. J. Am. Chem. Soc. 2019, 141 (2), 1054-1061. 
12. Walker, L. R.; Marty, M. T., Revealing the Specificity of a Range of Antimicrobial Peptides in Lipid Nanodiscs by Native Mass Spectrometry. Biochemistry 2020, 59 (23), 2135-2142.

13. Walker, L. R.; Marzluff, E. M.; Townsend, J. A.; Resager, W. C.; Marty, M. T., Native Mass Spectrometry of Antimicrobial Peptides in Lipid Nanodiscs Elucidates Complex Assembly. Anal. Chem. 2019, 91 (14), 9284-9291.

14. Li, J.; Han, L.; Li, J.; Kitova, E. N.; Xiong, Z. J.; Prive, G. G.; Klassen, J. S., Detecting Protein-Glycolipid Interactions Using CaR-ESI-MS and Model Membranes: Comparison of Pre-loaded and Passively Loaded Picodiscs. J. Am. Soc. Mass. Spectrom. 2018, 29 (7), 1493-1504.

15. Li, M. J.; Atkins, W. M.; McClary, W. D., Preparation of Lipid Nanodiscs with Lipid Mixtures. Curr. Protoc. Protein. Sci. 2019, 98 (1), e100.

16. Marty, M. T.; Zhang, H.; Cui, W.; Blankenship, R. E.; Gross, M. L.; Sligar, S. G., Native mass spectrometry characterization of intact nanodisc lipoprotein complexes. Anal. Chem. 2012, 84 (21), 8957-8960.

17. Marty, M. T.; Zhang, H.; Cui, W.; Gross, M. L.; Sligar, S. G., Interpretation and deconvolution of nanodisc native mass spectra. J. Am. Soc. Mass. Spectrom. 2014, 25 (2), 269-277.

18. El-Hawiet, A.; Shoemaker, G. K.; Daneshfar, R.; Kitova, E. N.; Klassen, J. S., Applications of a catch and release electrospray ionization mass spectrometry assay for carbohydrate library screening. Anal. Chem. 2012, 84 (1), 50-58

19. Han, L.; Kitov, P. I.; Li, J.; Kitova, E. N.; Klassen, J. S., Probing Heteromultivalent Protein-Glycosphingolipid Interactions using Native Mass Spectrometry and Nanodiscs. Anal. Chem. 2020, 92 (5), 3923-3931. 20. Han, L.; Morales, L. C.; Richards, M. R.; Kitova, E. N.; Sipione, S.; Klassen, J. S., Investigating the Influence of Membrane Composition on Protein-Glycolipid Binding Using Nanodiscs and Proxy Ligand Electrospray Ionization Mass Spectrometry. Anal. Chem. 2017, 89 (17), 9330-9338.

21. Cleary, S. P.; Prell, J. S., Distinct classes of multi-subunit heterogeneity: analysis using Fourier Transform methods and native mass spectrometry. Analyst 2020, 145 (13), 4688-4697.

22. Hoi, K. K.; Robinson, C. V.; Marty, M. T., Unraveling the Composition and Behavior of Heterogeneous Lipid Nanodiscs by Mass Spectrometry. Anal. Chem. 2016, 88 (12), 6199-6204.

23. Zhang, G.; Keener, J. E.; Marty, M. T., Measuring Remodeling of the Lipid Environment Surrounding Membrane Proteins with Lipid Exchange and Native Mass Spectrometry. Anal. Chem. 2020, 92 (8), 5666-5669.

24. Kostelic, M. M.; Ryan, A. M.; Reid, D. J.; Noun, J. M.; Marty, M. T., Expanding the Types of Lipids Amenable to Native Mass Spectrometry of Lipoprotein Complexes. J. Am. Soc. Mass. Spectrom. 2019, 30 (8), 1416 1425.

25. van Meer, G.; Voelker, D. R.; Feigenson, G. W., Membrane lipids: where they are and how they behave. Nat. Rev. Mol. Cell. Biol. 2008, 9 (2), $112-124$

26. Harayama, T.; Riezman, H., Understanding the diversity of membrane lipid composition. Nat. Rev. Mol. Cell. Biol. 2018, 19 (5), 281-296.

27. Xhindoli, D.; Pacor, S.; Benincasa, M.; Scocchi, M.; Gennaro, R.; Tossi, A., The human cathelicidin LL-37--A pore-forming antibacterial peptide and host-cell modulator. Biochim. Biophys. Acta. 2016, 1858 (3) 546-566.

28. Denisov, I. G.; Grinkova, Y. V.; Lazarides, A. A.; Sligar, S. G., Directed self-assembly of monodisperse phospholipid bilayer Nanodiscs with controlled size. J. Am. Chem. Soc. 2004, 126 (11), 3477-3487.

29. Marty, M. T.; Baldwin, A. J.; Marklund, E. G.; Hochberg, G. K. Benesch, J. L.; Robinson, C. V., Bayesian deconvolution of mass and ion mobility spectra: from binary interactions to polydisperse ensembles. Anal. Chem. 2015, 87 (8), 4370-4376.

30. Reid, D. J.; Diesing, J. M.; Miller, M. A.; Perry, S. M.; Wales, J. A.; Montfort, W. R.; Marty, M. T., MetaUniDec: High-Throughpu Deconvolution of Native Mass Spectra. J. Am. Soc. Mass. Spectrom. 2019, 30 (1), 118-127.
31. Horvath, S. E.; Daum, G., Lipids of mitochondria. Prog. Lipid. Res. 2013, 52 (4), 590-614

32. Corradi, V.; Sejdiu, B. I.; Mesa-Galloso, H.; Abdizadeh, H.; Noskov, S. Y.; Marrink, S. J.; Tieleman, D. P., Emerging Diversity in Lipid-Protein Interactions. Chem. Rev. 2019, 119 (9), 5775-5848.

33. van der Veen, J. N.; Kennelly, J. P.; Wan, S.; Vance, J. E.; Vance, D. E.; Jacobs, R. L., The critical role of phosphatidylcholine and phosphatidylethanolamine metabolism in health and disease. Biochim. Biophys. Acta. Biomembr. 2017, 1859 (9 Pt B), 1558-1572.

34. Brown, M. F., Curvature forces in membrane lipid-protein interactions. Biochemistry 2012, 51 (49), 9782-9795.

35. Townsend, J. A.; Keener, J. E.; Miller, Z. M.; Prell, J. S.; Marty, M T., Imidazole Derivatives Improve Charge Reduction and Stabilization for Native Mass Spectrometry. Anal. Chem. 2019, 91 (22), 14765-14772.

36. Arikketh, D.; Nelson, R.; Vance, J. E., Defining the importance of phosphatidylserine synthase-1 (PSS1): unexpected viability of PSS1deficient mice. J. Biol. Chem. 2008, 283 (19), 12888-12897.

37. Kay, J. G.; Fairn, G. D., Distribution, dynamics and functional roles of phosphatidylserine within the cell. Cell. Commun. Signal. 2019, 17 (1), 126.

38. Barenholz, Y.; Thompson, T. E., Sphingomyelin: biophysical aspects Chem. Phys. Lipids. 1999, 102 (1-2), 29-34.

39. Nyholm, T. K.; Nylund, M.; Slotte, J. P., A calorimetric study of binary mixtures of dihydrosphingomyelin and sterols, sphingomyelin, or phosphatidylcholine. Biophys. J. 2003, 84 (5), 3138-3146.

40. Filippov, A.; Oradd, G.; Lindblom, G., Sphingomyelin structure influences the lateral diffusion and raft formation in lipid bilayers. Biophys. J. 2006, 90 (6), 2086-2092.

41. Strahl, H.; Errington, J., Bacterial Membranes: Structure, Domains, and Function. Annu. Rev. Microbiol. 2017, 71 (1), 519-538.

42. Harvey, S. R.; VanAernum, Z. L.; Kostelic, M. M.; Marty, M. T.; Wysocki, V. H., Probing the structure of nanodiscs using surface-induced dissociation mass spectrometry. Chem. Commun. 2020, 56 (100), $15651-$ 15654.

43. Lopes, S.; Neves, C. S.; Eaton, P.; Gameiro, P., Cardiolipin, a key component to mimic the E. coli bacterial membrane in model systems revealed by dynamic light scattering and steady-state fluorescence anisotropy. Anal. Bioanal. Chem. 2010, 398 (3), 1357-1366.

44. El Khoury, M.; Swain, J.; Sautrey, G.; Zimmermann, L.; Van Der Smissen, P.; Decout, J. L.; Mingeot-Leclercq, M. P., Targeting Bacterial Cardiolipin Enriched Microdomains: An Antimicrobial Strategy Used by Amphiphilic Aminoglycoside Antibiotics. Sci. Rep. 2017, 7 (1), 10697 45. Paradies, G.; Paradies, V.; Ruggiero, F. M.; Petrosillo, G., Role of Cardiolipin in Mitochondrial Function and Dynamics in Health and Disease: Molecular and Pharmacological Aspects. Cells 2019, 8 (7).

46. Pizzuto, M.; Pelegrin, P., Cardiolipin in Immune Signaling and Cell Death. Trends Cell. Biol. 2020, 30 (11), 892-903.

47. Musatov, A.; Sedlak, E., Role of cardiolipin in stability of integral membrane proteins. Biochimie 2017, 142, 102-111.

48. Schlame, M.; Ren, M., The role of cardiolipin in the structural organization of mitochondrial membranes. Biochim. Biophys. Acta. 2009, 1788 (10), 2080-2083.

49. Mejia, E. M.; Hatch, G. M., Mitochondrial phospholipids: role in mitochondrial function. J. Bioenerg. Biomembr. 2016, 48 (2), 99-112.

50. Li, J.; Koh, J. J.; Liu, S.; Lakshminarayanan, R.; Verma, C. S.; Beuerman, R. W., Membrane Active Antimicrobial Peptides: Translating Mechanistic Insights to Design. Front. Neurosci. 2017, 11, 73. 
For TOC only:

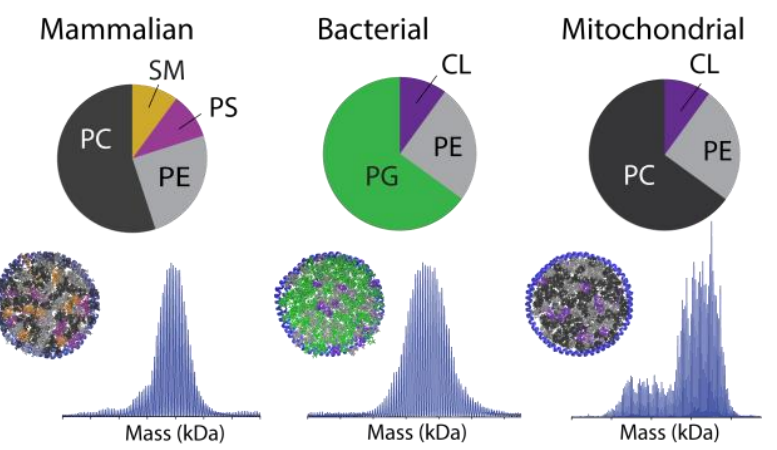

